\title{
Findings on Preoperative Brain MRI Predict Histopathology in Children with Cerebellar Neoplasms
}

\author{
Jonathan A. Forbes ${ }^{a}$ Adam S. Reig ${ }^{a}$ Jason G. Smith ${ }^{b}$ Walter Jermakowicz ${ }^{b}$ \\ Luke Tomycz $^{\text {a }}$ Sheila D. Shay ${ }^{a}$ David A. Sun ${ }^{d}$ Curtis A. Wushensky ${ }^{c}$ \\ Matthew M. Pearson ${ }^{a}$ \\ ${ }^{a}$ Department of Neurosurgery, ${ }^{b}$ Vanderbilt School of Medicine, and ${ }^{c}$ Department of Radiology and \\ Radiological Sciences, Vanderbilt University Medical Center, Nashville, Tenn., and d Department of \\ Neurosurgery, Norton Neuroscience Institute, Louisville, Ky., USA
}

\section{Key Words}

Magnetic resonance imaging $\cdot$ Cerebellar neoplasm •

Predicted histopathology $\cdot$ Pilocytic astrocytomas .

Medulloblastomas $\cdot$ Ependymomas

\begin{abstract}
Background/Aims: The majority of pediatric patients with cerebellar neoplasms harbor pilocytic astrocytomas (PAs), medulloblastomas, or ependymomas. Knowledge of a preoperative likelihood of histopathology in this group of patients has the potential to influence many aspects of care. Previous studies have demonstrated hyperintensity on diffusion-weighted imaging to correlate with medulloblastomas. Recently, measurement of $\mathrm{T}_{2}$-weighted signal intensity (T2SI) was shown to be useful in identification of low-grade cerebellar neoplasms. The goal of this study was to assess whether objective findings on these MRI sequences reliably correlated with the underlying histopathology. Methods: We reviewed the radiologic findings of 50 pediatric patients who underwent resection of a cerebellar neoplasm since 2003 at our institution. Region of interest placement was used to calculate the relative diffusion-weighted signal intensity (rDWSI) and relative T2SI (rT2SI) of each neoplasm. Results: Tukey's multiple comparison test demonstrated medulloblastomas
\end{abstract}

to have significantly higher rDWSIs than PAs/ependymomas, and PAs to have significantly higher rT2SIs than medulloblastomas/ependymomas. A simple method consisting of sequential measurement of rDWSI and $\mathrm{rT} 2 \mathrm{SI}$ to predict histopathology was then constructed. Using this method, 39 of 50 (78\%) tumors were accurately predicted. Conclusion: Measurement of rDWSI and rT2SI using standard MRI of the brain can be used to predict histopathology with favorable accuracy in pediatric patients with cerebellar tumors.

Copyright $\odot 2011$ S. Karger AG, Basel

\section{Introduction}

Overview

Intracranial tumors represent the most common solid tumor in the pediatric population [1], accounting for approximately 2,000 cases per year and an estimated 1 in 5 childhood cancers. Approximately $2 / 3$ of pediatric brain

A small portion of this work (relating to the ability of this scoring system to prevent wasteful spending on MRI) was presented at the 2009 Annual Meeting of the AANS/CNS Section on Pediatric Neurological Surgery, Boston, Mass., USA.

\section{KARGER}

(c) 2011 S. Karger AG, Basel

Fax +41613061234

E-Mail karger@karger.ch

www.karger.com
Accessible online at:

www.karger.com/pne
Jonathan A. Forbes

915 9th Avenue North

Nashville, TN 37208 (USA)

Tel. +1 6154162801

E-Mail jonathan.forbes@vanderbilt.edu 
tumors develop in the infratentorial compartment [2]. Within this infratentorial group of tumors, one can further differentiate between tumors which involve the brainstem and tumors which appear to be arising from the substance of the cerebellum, cerebellar peduncles, and/or fourth ventricle.

Pediatric cerebellar/fourth ventricular neoplasms are dominated by three disparate histopathologies - pilocytic astrocytoma (PA), medulloblastoma, and ependymoma - which together account for approximately $7 / 8$ of all tumors in this location. Magnetic resonance spectroscopy (MRS) has previously been demonstrated to be useful in differentiating among the three most common histopathologies of pediatric cerebellar tumors $[3,4]$. However, this method has been found to be of limited utility in the analysis of particularly small and/or cystic tumors. Furthermore, technical problems have been described in a significant proportion of patients analyzed. In the landmark paper detailing utilization of this method [3], only 30 of the 39 patients who underwent a complete MRS study were accepted for final analysis. Of this number, 22 ( $73 \%$ of those analyzed, but only $56 \%$ of those who underwent a complete study) were correctly identified as having an astrocytoma, ependymoma, or primitive neuroectodermal tumor (PNET). Of the 26 accepted patients found to harbor an astrocy toma, ependymoma, or PNET, $22(85 \%)$ were identified correctly. A more recent study involving a combination of diffusion-weighted imaging (DWI) and MRS in a relatively small series of patients demonstrated improved accuracy, but was unusually complex - requiring measurement of a total of seven variables [5]. Given the limitations described above - in addition to the general benefit of understanding the predictive value of various findings on standard imaging sequences routinely reviewed by the neurosurgery team analysis of the predictive value of findings on standard MRI was felt to be warranted.

The relationship between findings on standard MRI and histopathologic diagnosis of tumors arising from the substance of the cerebellum and/or fourth ventricle which together comprise approximately $50-55 \%$ of all pediatric intracranial tumors - remains vaguely defined. A literature search of radiologic findings thought to correlate with underlying histology in pediatric patients with cerebellar neoplasms yielded many case series [6-11], but no established method that was able to reliably identify the most likely histopathology based on MRI alone. Previous studies have demonstrated hyperintensity on DWI to be strongly predictive of medulloblastoma $[9,10]$. Recently, measurement of $\mathrm{T}_{2}$-weighted signal intensity
(T2SI) was shown to be useful in identification of lowgrade cerebellar neoplasms [12]. Because the preponderance of tumors in the cerebellar/fourth ventricular location fall into one of the aforementioned three histopathologic categories, the authors sought to assess whether objective findings on these MRI sequences could be used to accurately predict underlying histopathology.

\section{Clinical Materials and Methods}

\section{Radiologic Criteria and Patient Population}

Search of medical records at Vanderbilt Children's Hospital identified 58 patients with a cerebellar neoplasm who had undergone a craniotomy for primary resection since 2003. Because of initial MRI received at outside institutions, 7 of the 58 patients did not have diffusion-weighted sequencing and were excluded from the study. Another patient was excluded because on outside MRI, metallic orthodontic braces distorted the image significantly, confounding interpretation. Institutional review board approval and exemption status was obtained for the 50 patients who constituted the final study population. Conventional MRI was performed on all patients using a 1.5-T MR unit with the following sequences: sagittal non-contrast $\mathrm{T}_{1}$-weighted, axial fast spin-echo $\mathrm{T}_{2}$-weighted, axial fluid-attenuated inversion recovery (FLAIR), axial DWI, as well as post-contrast-enhanced axial, coronal, and sagittal $\mathrm{T}_{1}$-weighted imaging.

Using a PACS workstation, a pediatric neuroradiologist (C.A.W.) then conducted an independent, blinded assessment of the preoperative MR images with respect to the criteria listed above. Specifically, the relative diffusion-weighted signal intensity (rDWSI) and relative T2SI (rT2SI) were obtained in all patients. Scores assigned by the pediatric neuroradiologist (C.A.W.) were taken as final and used for all subsequent statistical analysis. Scores assigned in a blinded fashion by another author (W.J.) were analyzed in reference to the final scores documented by C.A.W. and used to assess interobserver reliability.

\section{Analysis of MRI Findings: Assessing Relative Diffusion}

Restriction

In order to assess the extent of diffusion restriction, the solid component of the cerebellar tumor is first located using axial $\mathrm{T}_{1^{-}}$ weighted imaging with contrast. In those rare tumors with sparse to no contrast enhancement, the solid component is instead located using $\mathrm{T}_{2}$-weighted fast spin-echo imaging. The intensity of the solid component of the tumor is then evaluated in relation to normal adjacent cerebellar gray and white matter on DWI. ADC mapping of the lesion is referenced against DWI to ensure that ' $\mathrm{T}_{2}$ shine-through' is not responsible for factitious DWI hyperintensity - this is accomplished by ensuring that areas of solid component found to be hyperintense on DWI correspond to areas of hypointensity on ADC mapping. Once an appropriate axial diffusion-weighted image with relatively generous visible areas of neoplasm and normal cerebellar gray/white matter is found (see fig. 1), a region of interest (ROI) is superimposed on a representative portion of the solid component and the signal intensity within is quantitated in PACS. Whenever possible, the area of the ROI was targeted to a value of at least $0.5 \mathrm{~cm}^{2}$ when limited to the avail- 
able solid component. For tumors, such as high-grade nonPNETs, which exhibit considerable imaging heterogeneity, the ROI is superimposed on a slice with a solid component signal representative of the majority of tumor. After tumor DWI intensity has been assessed, this action is then repeated with normal, lateral cerebellar gray matter as illustrated below (midline, suboccipital gray matter is avoided as it is often spuriously hyperintense on DWI). The area of this ROI is sometimes comparatively smaller than the area of the ROI used to measure signal intensity of the neoplasm. Of note, normal cerebellar gray matter is routinely more hyperintense on standard DWI than normal cerebellar white matter; this property is used to help guide ROI placement. It is essential to insure that all ROIs - whether pertaining to cerebellar gray matter or neoplasm - contain only the target substrate within their borders.

After a numerical value specifying the intensity of the solid component of the neoplasm on DWI has been obtained, it is then divided by the diffusion-weighted intensity of normal, cerebellar gray matter for purposes of normalization. The resultant value is termed the relative diffusion-weighted signal intensity (rDWSI). Describing the diffusion-weighted intensity of tumor relative to normal cerebellar gray matter in this manner permits some degree of standardization and allows for manageable future comparison of data. Following radiologic evaluation, neoplasms are stratified as DWI hyperintense (rDWSI $>1$ ) or DWI hypointense (rDWSI $\leq 1$ ) accordingly.

The vast majority of DWI-hyperintense cerebellar neoplasms are WHO grade IV lesions - most of these being medulloblastomas. The vast majority of ependymomas and PAs are DWI hypointense neoplasms. An example of the corresponding diffusion-weighted signal intensities of the three histopathologies is found in figure 2 .

\section{Analysis of MRI Findings: Assessing Relative $T_{2}$ Signal \\ Intensity}

We have previously described our method for evaluating the rT2SI of cerebellar neoplasms in a publication describing its utility in identifying tumors at an elevated risk for leptomeningeal dissemination [12]. Briefly, the measurement is as follows: the solid component of the tumor in question is again identified. A $\mathrm{ROI}$ is then placed on a representative portion of the solid component and the signal intensity is quantitated using PACS software (see fig. 3). For purposes of normalization, a ROI is also superimposed on vitreous humor as illustrated, where signal intensity of vitreous is measured. The rT2SI of the tumor - defined as the numerical value indicative of the degree of T2SI of solid tumor divided by the numerical value indicative of the degree of T2SI of vitreous humor - is then determined. If the relative $\mathrm{T}_{2}$ intensity is $>0.71$, the tumor is considered to be $\mathrm{T}_{2}$ hyperintense - strongly indicative of PAs. If the relative $\mathrm{T}_{2}$ intensity is $\leq 0.71$, the tumor is considered to be $\mathrm{T}_{2}$ hypointense. An example of the corresponding T2SIs of a medulloblastoma and a PA is found in figure 4 .

\section{Statistical Considerations}

Statistical analyses were performed in GraphPad Prism 5.03 (GraphPad Software Inc., La Jolla, Calif., USA). Datasets of rDWSI and rT2SI obtained from the 42 patients with PA, medulloblastoma, or ependymoma were grouped according to histopathology. Data from the 8 remaining patients with other various

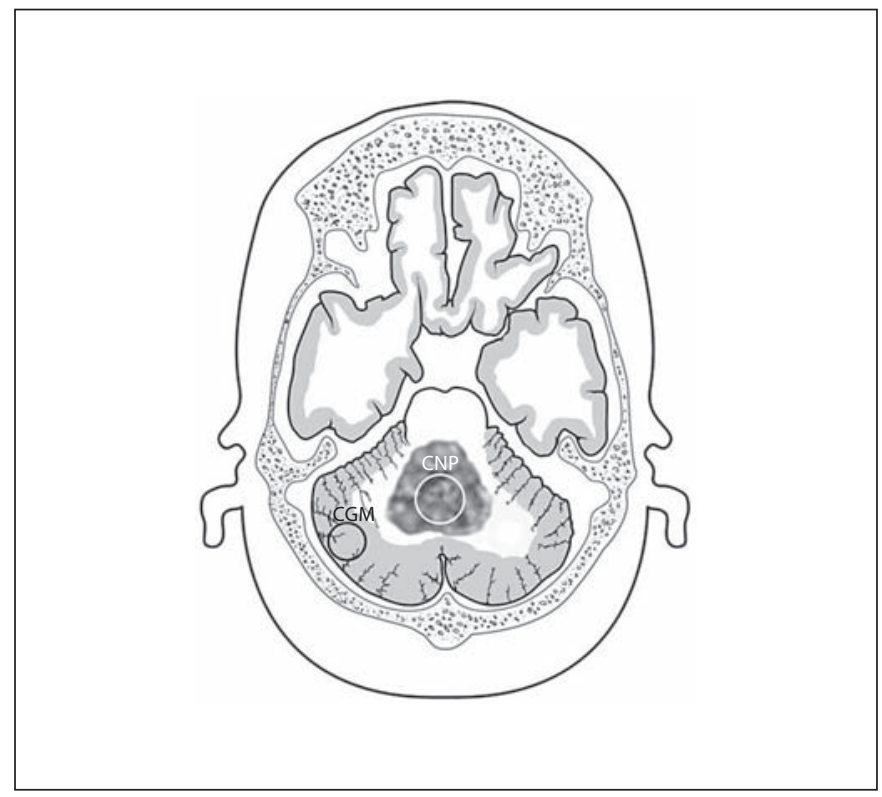

Fig. 1. Depiction of cerebellar neoplasm: regions of comparison for DWI mapping are illustrated. The diffusion-weighted intensity of the solid component of the tumor is estimated by measuring the signal intensity of a ROI (CNP). rDWSI is obtained by dividing the DWSI of solid tumor (CNP) by the DWSI of cerebellar gray matter (CGM). If the DWI signal of the neoplasm is brighter than that of CGM (rDWSI $>1$ ), the tumor is stratified as DWI hyperintense. If the DWI signal is equal or less than that of CGM (rDWSI <1), the tumor is classified as DWI hypointense.

histopathologies was not included in the analysis. One-way analysis of variance (ANOVA) was first used to assess if a difference was present between two or more of the groups. Subsequent analysis with Tukey's multiple comparison test was then used to assess for a significant difference among the individual groups (PA vs. medulloblastoma, medulloblastoma vs. ependymoma, ependymoma vs. PA). A p value $<0.05$ was considered statistically significant.

Interobserver reliability for the methods of attainment of rDWSI and rT2SI were subsequently assessed. The initial dataset used for the above-mentioned analysis had been constructed using data obtained by an attending pediatric neuroradiologist. This dataset was then referenced against rDWSI and rT2SI data obtained in a blinded manner by a third-year medical student to evaluate interobserver reliability. The medical student had received approximately $30 \mathrm{~min}$ of training in how to measure the aforementioned parameters. Using these two numerical datasets, interobserver reliability of the rDWSI and rT2SI was assessed using the Spearman rank correlation coefficient. Additionally, nominal categories of the datasets (rDWSI: DWI hyperintense, DWI hypointense; rT2SI: $\mathrm{T}_{2}$ hyperintense, $\mathrm{T}_{2}$ hypointense) were assessed via calculation of the Cohen unweighted $\kappa$ coefficient. The $\kappa$ coefficient measures agreement between observers beyond that expected by chance alone [13]. 

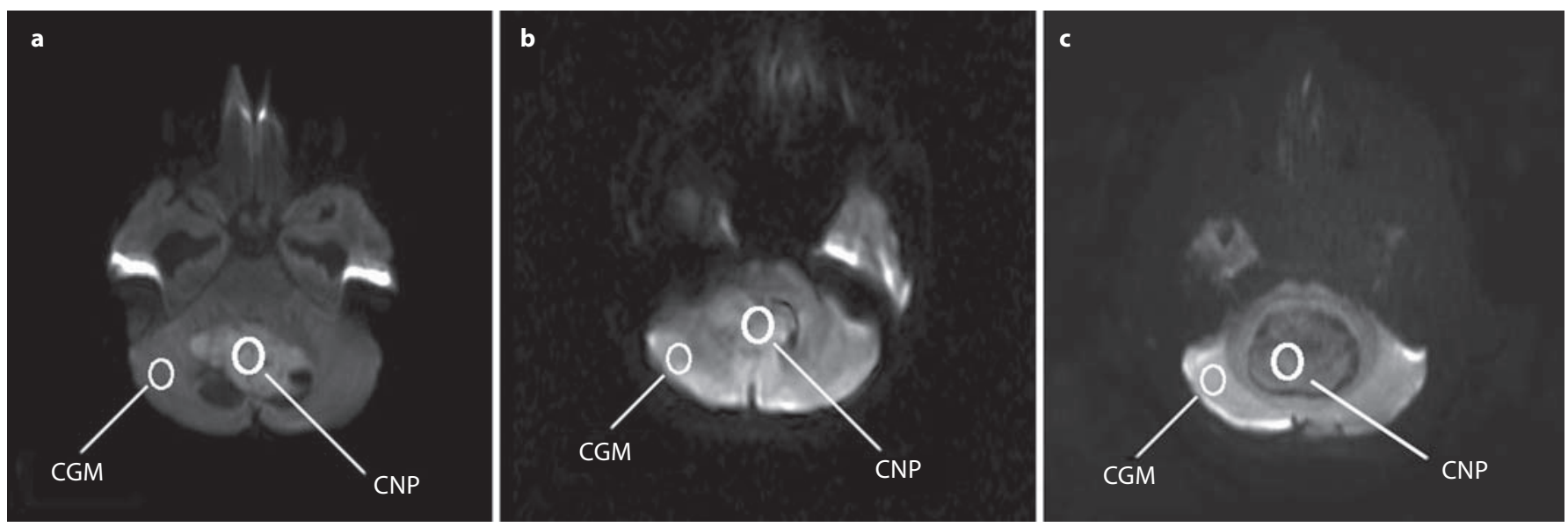

Fig. 2. Images demonstrating the diffusion-weighted characteristics of different histopathologies. The midline cerebellar neoplasms are: a medulloblastoma (DWI hyperintense), b ependymoma (DWI hypointense), and c PA (DWI hypointense). The rDWSIs of medulloblastoma are significantly greater than those

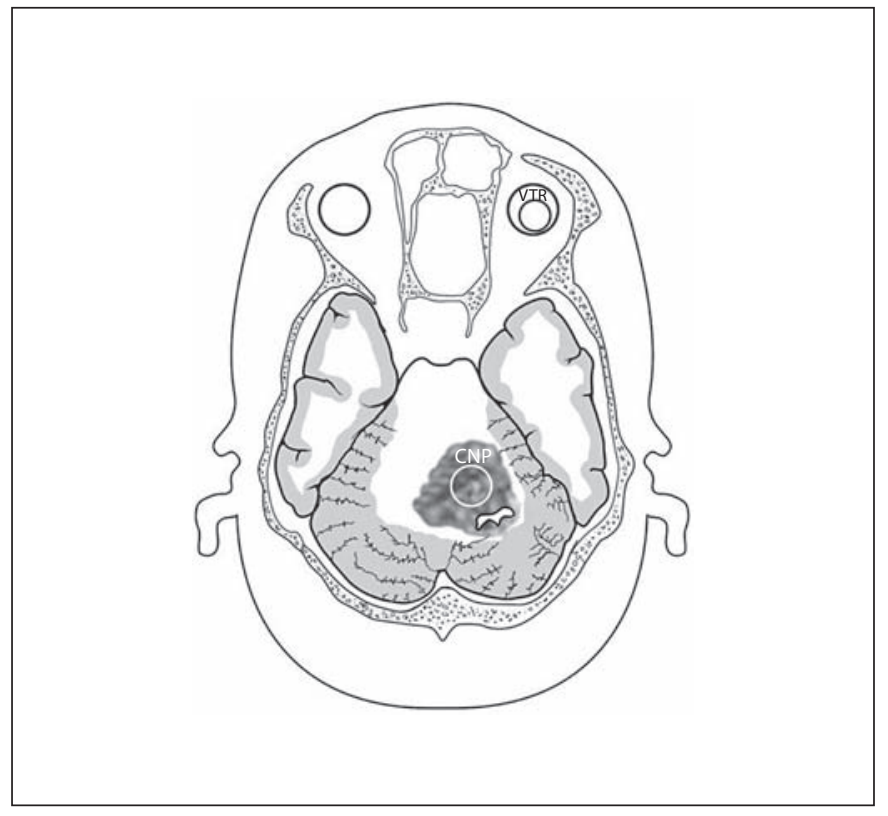

Fig. 3. Depiction of cerebellar neoplasm: regions of comparison for $\mathrm{T}_{2}$-weighting. The T2SI of the solid component of the tumor is estimated by measuring the signal intensity of a ROI (CNP) on a standard axial $\mathrm{T}_{2}$-weighted image. This is compared to a value obtained from a ROI involving normal vitreous humor (VTR). The rT2SI is obtained by dividing the T2SI of solid tumor by the T2SI of normal vitreous. If this value is $>0.71$, the tumor is designated as $T_{2}$ hyperintense. Otherwise, the tumor is designated as $\mathrm{T}_{2}$ hypointense. of ependymomas and PAs. While the rDWSIs of ependymomas are often slightly greater than the rDWSIs of PAs, this difference did not reach statistical significance. CGM = ROI selected to measure DWI signal intensity of cerebellar gray matter; CNP = ROI selected to measure DWI signal intensity of cerebellar neoplasm.

\section{Results}

One-way ANOVA indicated that a significant difference $(p<0.05)$ was present among the three groups in both (rDWSI and rT2SI) datasets. Using Tukey's multiple comparison test, medulloblastomas were found to have rDWSIs significantly higher (95\% CI 1.13-1.36) than both ependymomas (95\% CI $0.55-1.07)$ and PAs (95\% CI 0.53-0.72). The difference between the rDWSIs of ependymomas and PAs was not statistically significant. A plot of the rDWSIs utilized in this analysis, grouped by histopathology, is depicted in figure 5.

Additionally, the rT2SIs of the three major histopathologies were analyzed using Tukey's multiple comparison test. Using this method of analysis, PAs were found to have significantly higher rT2SIs (95\% CI 0.82-0.95) than both ependymomas (95\% CI 0.37-0.52) and medulloblastomas (95\% CI 0.48-0.58). The difference between the rT2SIs of ependymomas and medulloblastomas did not reach statistical significance. A plot of the rT2SIs utilized in this analysis, grouped by histopathology, is depicted in figure 6 .

As previously mentioned, assessment of interobserver reliability of methods of attainment of the rDWSI and rT2SI was computed by comparing data obtained by an attending pediatric neuroradiologist to data obtained by a third-year medical student. The Spearman rank correlation coefficient between the rDWSI data of the two observers was 0.971 (95\% CI 0.949-0.983, p < 0.001), and for 

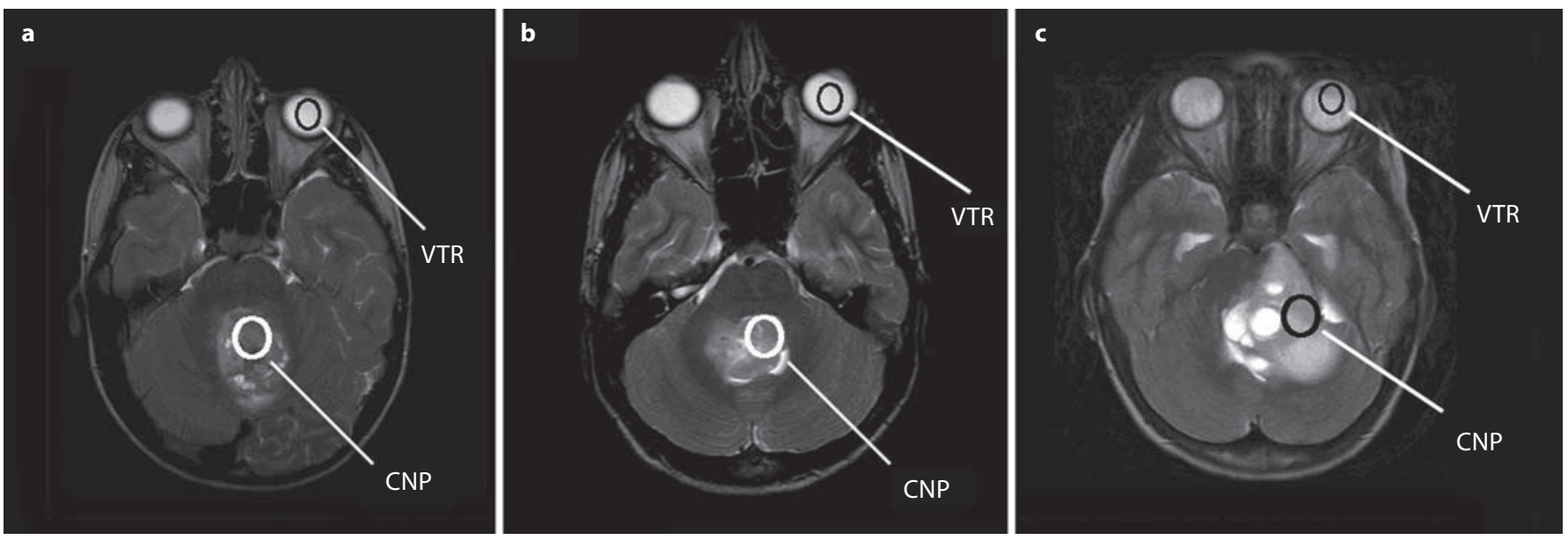

Fig. 4. Images demonstrating the $\mathrm{T}_{2}$-weighted characteristics of different histopathologies. The midline cerebellar neoplasms are: a medulloblastoma ( $\mathrm{T}_{2}$ hypointense), b ependymoma ( $\mathrm{T}_{2}$ hypointense), and c PA ( $\mathrm{T}_{2}$ hyperintense). The average T2SI of PAs (approx. as bright as vitreous humor, in this example) is signifi- cantly higher than that of medulloblastomas and ependymomas. VTR = Vitreous, represents ROI selected to measure T2SI of vitreous; $\mathrm{CNP}=$ cerebellar neoplasm, represents ROI selected to measure T2SI of the solid component of CNP.

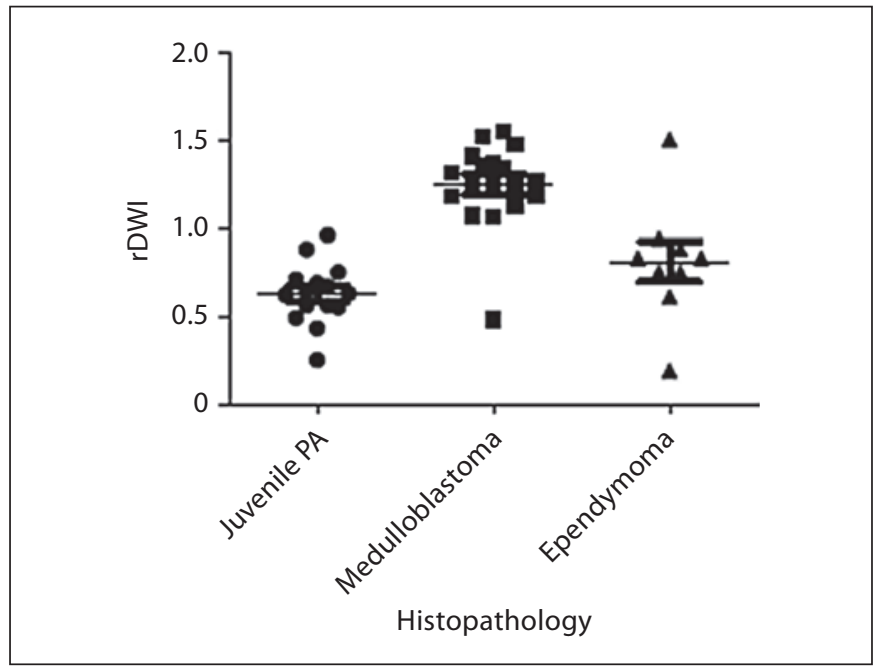

Fig. 5. Relative diffusion-weighted intensities (rDWIs) of cerebellar neoplasms stratified by histopathology. Using Tukey's multiple comparison test to analyze this data, medulloblastomas were found to have rDWSIs significantly higher than both ependymomas and PAs.

the rT2SI data it was 0.967 (95\% CI 0.941-0.981, p < 0.001). The Cohen unweighted $\kappa$ coefficient for the two observers was calculated to compare the interobserver reliability of nominal designations. In general, higher $\kappa$ coefficients indicate a greater degree of agreement between two observers - with values $>0.80$ indicating a high degree of agree-

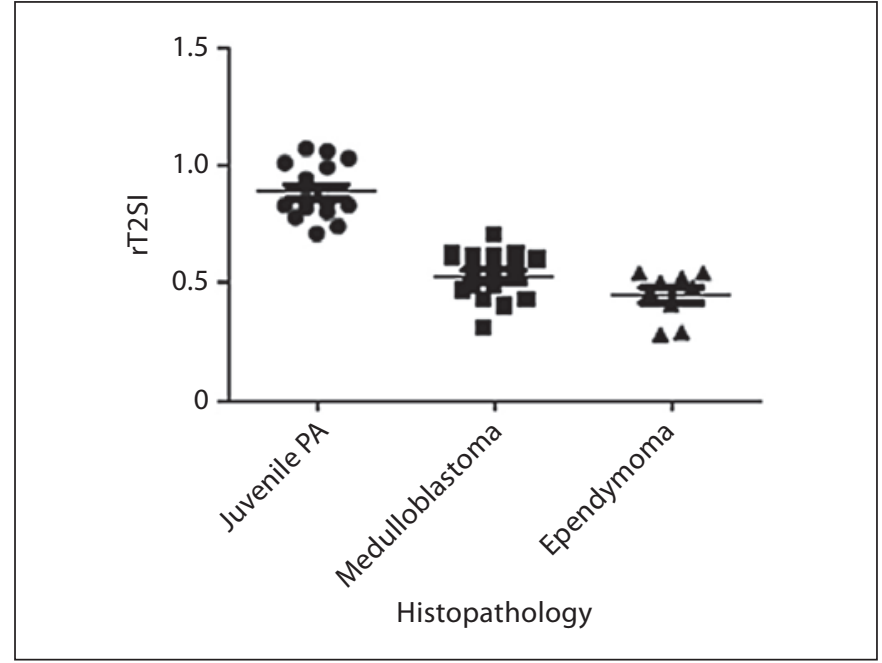

Fig. 6. rT2SIs of cerebellar neoplasms stratified by histopathology. Using Tukey's multiple comparison test to analyze this data, PAs were found to have significantly higher rT2SIs than both ependymomas and medulloblastomas (95\% CI 0.48-0.58).

ment. Data between the two observers were highly correlated. The two observers predicted the same pathology in $49 / 50$ cases, corresponding to a $\kappa$ coefficient of 0.969 ( $95 \%$ CI $0.909-1.000, \mathrm{p}<0.001)$. These data indicate near-identical correspondence between the raters - suggesting this method can be used among raters of variable experience. 
Fig. 7. Decision tree describing the Vanderbilt method for determination of likelihood of pediatric cerebellar neoplasms. Of note, 17 of 18 medulloblastomas were DWI hyperintense; 8 of 9 ependymomas were DWI hypointense/ $\mathrm{T}_{2}$ hypointense; 14 of 15 PAs were DWI hypointense/ $\mathrm{T}_{2}$ hyperintense.

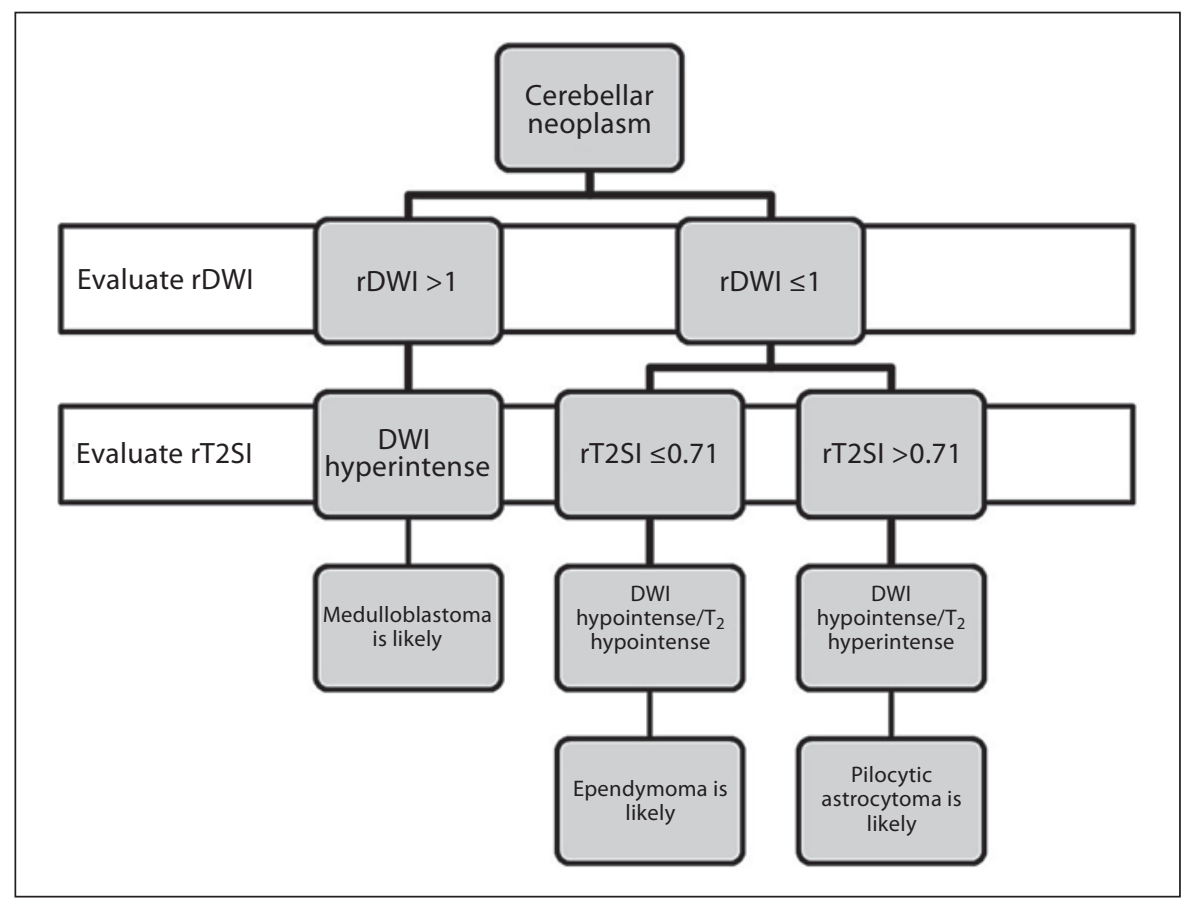

Table 1. Relative diffusion-weighted intensity stratified by histopathology

\begin{tabular}{lccl}
\hline Histopathology & $\begin{array}{l}\text { DWI hyper- } \\
\text { intense }\end{array}$ & $\begin{array}{l}\text { DWI hypo- } \\
\text { intense }\end{array}$ & $\begin{array}{l}\text { Avg. Rel. } \\
\text { DWI }\end{array}$ \\
\hline Medulloblastoma & 17 & 1 & 1.25 \\
PA & 0 & 15 & 0.63 \\
Ependymoma & 1 & 8 & 0.81 \\
PMA & 0 & 2 & 0.73 \\
Other, grade I & 0 & 2 & 0.50 \\
Other, grade II & 0 & 2 & 0.72 \\
Other, grade IV & 1 & 1 & 0.95 \\
\hline Total & 19 & 31 & \\
\hline
\end{tabular}

$\mathrm{PA}=$ Pilocytic astrocytoma; PMA = pilomyxoid astrocytoma; Avg. Rel. DWI = corresponds to averaging of the relative DWI signals (DWI signal of tumor/DWI signal cerebellar white matter) for the corresponding histopathologies.

Criterion 1: Relative Diffusion-Weighted Signal Intensity In this series of 50 patients -42 of whom harbored medulloblastomas, PAs, or ependymomas - classification of a lesion as DWI hyperintense (rDWSI $>1$ ) was $94 \%$ specific and 94\% sensitive for the detection of medulloblastoma. All but 1 of the 18 patients with medulloblastomas demonstrated DWI hyperintensity (table 1). The sole pa- tient with a histopathologic diagnosis of medulloblastoma without evidence of a DWI-hyperintense lesion on MRI had suffered significant hemorrhage involving the entirety of the tumor. In addition to the 17 patients with medulloblastoma, 2 other patients harbored lesions found to be DWI hyperintense: 1 of these was a 'poorly unspecified grade IV lesion', while the other harbored a grade III ependymoma. Eighteen of 19 patients with lesions that demonstrated DWI hyperintensity were found to harbor grade IV lesions. Notably, one grade IV GBM did not show evidence of DWI hyperintensity.

\section{Criterion 2: Relative $T_{2}$ Signal Intensity}

Independent classification of a lesion as $\mathrm{T}_{2}$ hyperintense was $86 \%$ specific and $93 \%$ sensitive for the detection of PA. Fourteen of 15 PAs exhibited $\mathrm{T}_{2}$ hyperintensity; the lone PA that was not $\mathrm{T}_{2}$ hyperintense had a rT2SI of 0.71 - a value equal to the threshold level. This PA exhibited the classic 'mural nodule' appearance, with an extremely small solid component. In addition to the $14 \mathrm{pa}-$ tients with $\mathrm{T}_{2}$-hyperintense PAs, 5 other patients harbored $\mathrm{T}_{2}$-hyperintense lesions - including 2 PMAs, 2 grade I tumors, and 1 grade II tumor (table 2).

\section{Final Analysis of Criteria}

Following demonstration of statistically significant differences in radiologic findings described in the sec- 
tions above, a method for determining the histopathologic likelihood of pediatric cerebellar neoplasms was devised (fig. 7). Using this method, histopathologic likelihood can be assessed following simple calculations of rDWSI and rT2SI.

Using retrospective application of the method detailed in figure 7, 14 of 15 PAs, 8 of 9 ependymomas, and 17 of 18 medulloblastomas would have been predicted correctly. Of the 42 patients who harbored PAs, medulloblastomas, or ependymomas, 39 would be predicted correctly (93\%). Of the 50 patients who presented with cerebellar neoplasms (table 3), the correct histopathology would have retrospectively been predicted in 39 patients (78\%).

\section{Discussion}

Histologic Variants: Radiologic Findings,

\section{Contemporary Therapy}

PAs comprise approximately $1 / 3$ of pediatric cerebellar neoplasms and $80-85 \%$ of cerebellar astrocytomas [14]. This subset of neoplasms is associated with a favorable prognosis - with an estimated $94 \%$ 10-year survival rate [15]. There are certain criteria on standard MRI sequences that are somewhat unique to these neoplasms. First, the origin of PAs in the cerebellum is variable. Unlike ependymomas and most pediatric medulloblastomas, they often originate from the cerebellar hemispheres and/or peduncles. Furthermore, relative to ependymomas and medulloblastomas, PAs are generally more hyperintense on $\mathrm{T}_{2}$-weighted imaging [7]. This finding is most likely attributable to characteristic histopathologic features which include a loose, microcystic background [14]. Because of the relatively sparse cellularity of these lesions, PAs also tend to demonstrate less restricted diffusion than ependymomas and medulloblastomas, and are hypointense on DWI relative to these other histopathologies [10]. In relation to adjacent cerebellar tissue, PAs are hypointense to cerebellar gray matter and are usually hypointense in relation to cerebellar white matter on DWI.

In contrast to PAs, medulloblastomas - which represent approximately $3 / 8$ of pediatric cerebellar neoplasms - are biologically aggressive neoplasms. Children with medulloblastoma are traditionally divided into standard and high-risk groups based on the presence or absence of risk factors. High-risk children are younger than 3 years, have evidence of leptomeningeal spread or systemic dissemination, or are noted to have residual tumor of $>1.5 \mathrm{~cm}^{2}$ following resection. Survival rates of pa-
Table 2. Relative T2-weighted signal intensity stratified by histopathology

\begin{tabular}{lccl}
\hline Histopathology & $\begin{array}{l}\text { T2 hyper- } \\
\text { intense }\end{array}$ & $\begin{array}{l}\text { T2 hypo- } \\
\text { intense }\end{array}$ & $\begin{array}{l}\text { Avg. Rel. T2 } \\
\text { intensity }\end{array}$ \\
\hline Medulloblastoma & 0 & 18 & 0.53 \\
PA & 14 & 1 & 0.89 \\
Ependymoma & 0 & 9 & 0.45 \\
PMA & 2 & 0 & 0.81 \\
Other, grade I & 2 & 0 & 0.78 \\
Other, grade II & 1 & 1 & 0.81 \\
Other, grade IV & 0 & 2 & 0.53 \\
\hline Total & 21 & 29 &
\end{tabular}

Avg. Rel. T2 intensity corresponds to averaging of the relative T2 intensities for the corresponding histopathology. Relative T2 intensity $=\mathrm{T} 2$ intensity tumor/T2 intensity vitreous.

Table 3. Results indicating how predicted histopathology correlated with actual histopathology

\begin{tabular}{lcrcc}
\hline \multirow{2}{*}{$\begin{array}{l}\text { Actual } \\
\text { histopathology }\end{array}$} & $\begin{array}{l}\text { Number } \\
\text { of } \\
\text { cases }\end{array}$ & \multicolumn{3}{c}{ Predicted histopathology } \\
\cline { 3 - 5 } & 15 & 14 & 1 & 0 \\
PA ependy- & $\begin{array}{l}\text { medullo- } \\
\text { moma }\end{array}$ & & 1 \\
PA & 9 & 0 & 8 & 17 \\
Ependymoma & 18 & 0 & 1 & 0 \\
Medulloblastoma & 2 & 2 & 0 & 0 \\
PMA & 2 & 2 & 0 & 1 \\
Other, grade I & 4 & 1 & 2 & 19 \\
Other, grade II-IV & 50 & 19 & 12 & \\
\hline Total & & &
\end{tabular}

tients affected with these lesions have improved significantly over the past 30 years, with recent treatment strategies in standard-risk patients producing 5-year survival rates surpassing $80 \%$ [16] and more aggressive protocols in patients with high-risk disease resulting in 5-year survival rates ranging from 60 to $70 \%$ [17]. In contrast to PAs, pediatric medulloblastomas are often midline with demonstrated involvement of the vermis and/or fourth ventricle and variable extension into the hemispheres [19]. Due to elevated tumor cellularity and an increased proportion of nuclear area, medulloblastomas demonstrate more restricted diffusion of water than ependymomas and astrocytomas, and are hyperintense on DWI relative to these other histologies. In relation to adjacent cerebellar tissue, medulloblastomas are consistently hyperintense to cerebellar gray and white matter on DWI [10]. 
Although tumor exit through the outlet foramina of the fourth ventricle does sometimes occur, medulloblastomas are much less likely than ependymomas to exhibit this phenomenon [8]. Patients with medulloblastoma have elevated associated risks of leptomeningeal dissemination at time of presentation [12]. Preoperative knowledge of whether or not leptomeningeal spread is present in patients suspected to harbor medulloblastoma is useful to the pediatric neurosurgeon, as this can influence whether or not the patient is deemed 'high risk' and concomitantly alter the risk/benefit analysis of aggressive surgical resection in eloquent locations.

Ependymomas, which comprise nearly $1 / 8$ of pediatric cerebellar tumors, typically arise in the vicinity of the fourth ventricle. Although frequently subcategorized as either grade II or grade III (anaplastic) lesions, grade has not been shown to affect mortality or clinical behavior [1]. Overall 5- and 10-year survival rates in children with ependymoma are approximately 60 and 45\%, respectively. Ependymomas are intermediate with respect to signal intensity on DWI in relation to that of medulloblastomas and PAs. Their signal on MRI is typically somewhat heterogeneous with respect to this imaging sequence, but peak DWI signal intensity reliably ranges from slightly hyperintense to cerebellar white matter to isointense with cerebellar gray matter. Cerebellar ependymomas involve the fourth ventricle and usually extend to some extent through the outlet foramina of Magendie and Luschka [18]. In light of an elevated risk of leptomeningeal dissemination [12], preoperative imaging of the neuraxis in patients suspected to harbor ependymoma is recommended. As chemotherapy has not been shown to significantly improve outcome in patients with ependymoma, surgical resection and postoperative irradiation of the primary site (dose has traditionally ranged from 4,500 to 5,600 cGy) have remained the mainstays of therapy. Extent of resection is the factor most strongly associated with prognosis. Progression-free survival rates after complete resection generally approximate $70-80 \%$, compared to less than $35 \%$ after incomplete resection [1]. Because gross total surgical resection is such a vital prognostic factor in the pediatric patient with ependymoma, preoperative knowledge of likelihood of ependymoma is useful to the pediatric neurosurgeon.

\section{Imaging Protocols: Correlation with Final \\ Histopathologic Diagnosis}

In DWI, phase-defocusing and re-focusing gradients are used to evaluate the restricted diffusion of water within tissue. DWI is ideal for use in a population of patients with location-specific neoplasms where there is great disparity regarding cellularity and proportion of nuclear area amongst the predominant histologies and has proven essential in analyzing the pediatric patient with a cerebellar tumor [10]. Regarding diffusion weighting as it relates to the predominant pathologies of pediatric cerebellar tumors, the following generalizations can be made: medulloblastomas are more hyperintense on DWI than ependymomas, which in turn appear to be slightly more hyperintense on DWI than PAs. In our study, fulfilling the criteria for DWI hyperintensity was extremely sensitive and specific for the detection of medulloblastoma. However, this parameter was not useful for differentiation of ependymomas from astrocytomas.

While the assessment of rDWSI is able to identify medulloblastomas with high precision, the second criterion of rT2SI was selected to aid in the differentiation of ependymomas from astrocytomas. In general, PAs demonstrate marked hyperintensity of the solid component of tumor on $\mathrm{T}_{2}$-weighted imaging. In an analysis by Arai et al. [7] in 2006, 5 of 10 patients (50\%) with PA displayed $\mathrm{T}_{2}$-weighted signals isointense to CSF. In a previous study, we found that we could increase the sensitivity of this finding without altering the specificity by describing the intensity on $\mathrm{T}_{2}$-weighted imaging of the tumor in relation to the $\mathrm{T}_{2}$-weighted signal of vitreous humor [12]. Specifically, if the $\mathrm{T}_{2}$-weighted signal of the solid component of the tumor was greater than $71 \%$ of the signal of vitreous humor, the tumor was deemed $\mathrm{T}_{2}$ hyperintense - a parameter shown to be accurate for identification of low-grade tumors with a decreased risk of leptomeningeal dissemination. By routinely assessing this parameter, preoperative imaging of the neuraxis in patients with low-grade tumors and an extremely low preoperative risk of leptomeningeal metastasis could be avoided [12]. In the present analysis, independent use of the rT2SI parameter demonstrated $\mathrm{T}_{2}$ hyperintensity $(\mathrm{rT} 2 \mathrm{SI}>0.71)$ to be $86 \%$ specific and $93 \%$ sensitive for a diagnosis of PA.

When interpreting the radiologic findings of posterior fossa tumors, it is common for pediatric neuroradiologists to include an assessment of the most likely underlying histopathology. Accordingly, retrospective analysis revealed that Vanderbilt neuroradiologists correctly anticipated histopathology in 35 of 48 (73\%) of the patients in this study (no prediction was provided for 2 patients). Among the remaining 13 patients who received inaccurate preoperative diagnoses, 7 were ultimately diagnosed with ependymoma, PA, or medulloblastoma. Using the objective criteria of the Vanderbilt method described in this paper, $78 \%$ of histopathologies were correctly pre- 
dicted. Of the 11 patients in which the histopathology was not accurately predicted, only 3 harbored ependymomas, PAs, or medulloblastomas. In addition, many of the 11 patients were found to have actual histopathologies of identical grade to predicted histopathology. Thus, the simple and objective method presented in this paper appears to compare favorably with the predictions of experienced neuroradiologists.

\section{Limitations}

A significant limitation of the Vanderbilt method described in this paper is the inability to predict the likelihood of histopathologies other than ependymomas, medulloblastomas, or PAs. A second potential limitation is the uncharacterized variation in DWI signal intensity of cerebellar gray matter and T2SI of vitreous humor - both of which were chosen in this study for normalization of respective parameters. Obviating the need to measure signal intensities of cerebellar gray matter and vitreous humor would be expected to expedite the time to data acquisition and improve predictive value. Currently, however, no preselected MRI parameters exist which can be referenced against the expected DWI and T2SIs of the different histopathologies, necessitating the previously described method for standardization. Another limitation of this study is that intraobserver reliability was not assessed. One final limitation of this study is that it is a retrospective analysis. Future characterization of the aforementioned data via prospective methods should follow.

\section{Conclusion}

In this study, measurement of the rDWSI and rT2SI was used to predict histopathology in pediatric patients with cerebellar neoplasms. The method is as follows: in tumors found to exhibit a rDWSI $>1$, medulloblastoma is suspected. In tumors with a $\mathrm{rDWSI} \leq 1$, the rT2SI is measured. In this group, if the rT2SI is $\leq 0.71$, ependymoma is suspected. If the rT2SI is $>0.71$, PA is suspected. Using this method, 39 of 50 (78\%) tumors were accurately predicted - including 14 of 15 PAs, 8 of 9 ependymomas, and 17 of 18 medulloblastomas. This method was found to have high interobserver reliability on statistical analysis.

\section{Acknowledgement}

This study was supported in part by Vanderbilt CTSA grant UL1 RR024975 from NCRR/NIH.

\section{References}

1 Kun LE: Brain tumors: challenges and directions. Pediatr Clin North Am 1997;44:907917.

-2 Nejat F, Khashab M, Rutka J: Initial management of childhood brain tumors: neurosurgical considerations. J Child Neurol 2008;23: 1136-1148.

-3 Wang Z, Sutton LN, Cnaan A, Haselgrove JC, Rorke LB, Zhao H, Bilaniuk LT, Zimmerman RA: Proton MR spectroscopy of pediatric cerebellar tumors. Am J Neuroradiol 1995; 16:1821-1833.

4 ZakrzewskiK, KreiselJ,Polis L, Nowosławska E, Liberski PP, Biegański T: Clinical application of proton magnetic resonance spectroscopy for differential diagnosis of pediatric posterior fossa tumors. Neurol Neurochir Pol 2001;5(suppl):19-25

5 Schneider JF, Viola A, Confort-Gouny S, Ayunts K, Le Fur Y, Viout P, Bennathan M, Chapon F, Figarella-Branger D, Cozzone P, Girard N: Infratentorial pediatric brain tumors: the value of new imaging modalities. J Neuroradiol 2007;34:49-58

6 Abdelhalim AN, Alberico RA: Pediatric neuroimaging. Neurol Clin 2009;27:285-301.
-7 Arai K, Sato N, Aoki J, Yagi A, TaketomiTakahashi A, Morita H, Koyama Y, Oba H, Ishiuchi S, Saito N, Endo K: MR signal of the solid portion of pilocytic astrocytoma on $\mathrm{T}_{2-}$ weighted images: is it useful for differentiation from medulloblastoma? Neuroradiology 2006;48:233-237.

-8 Blaser SI, Harwood-Nash DC: Neuroradiology of pediatric posterior fossa medulloblastoma. J Neurooncol 1996;29:23-34.

$\checkmark 9$ Chawla A, Emmanuel JV, Seow WT, Lou J, Teo HE, Lim CC: Paediatric PNET: pre-surgical MRI features. Clin Radiol 2007;62:43-52.

10 Rumboldt Z, Camacho DL, Lake D, Welsh CT, Castillo M: Apparent diffusion coefficients for differentiation of cerebellar tumors in children. Am J Neuroradiol 2006;27: 1362-1369.

11 Trasimeni G, Lenzi J, Di Biasi C, Anichini G, Salvati M, Raco A: Midline medulloblastoma versus astrocytoma: the position of the superior medullary velum as a sign for diagnosis. Childs Nerv System 2008;24:1037-1041.

12 Forbes JA, Chambless LB, Smith JG, Wushensky CA, Lebow RL, Alvarez J, Pearson MM: Use of $T_{2}$ signal intensity of cerebellar neoplasms in pediatric patients to guide preoperative staging of the neuraxis. J Neurosurg Pediatr 2011;7:165-174.

13 Landis JR, Koch GG: The measurement of observer agreement for categorical data. Biometrics 1977;33:159-174.

14 Lo S, Kish K, Chang E, Levin K, Keole S, Sloan A, Fontanesi J: Juvenile pilocytic astrocytoma. eMed J 2004;3:1-13.

15 Koeller KK, Rushing EJ: From the archives of the AFIP: pilocytic astrocytoma: radiologicpathologic correlation. Radiographics 2004; 24:1693-1708.

16 Pollack IF: Pediatric brain tumors. Semin Surg Oncol 1999;16:73-90.

17 Packer RJ: Childhood brain tumors: accomplishments and ongoing challenges. J Child Neurol 2008;23:1122.

18 Tortori-Donati P, Fondelli MP, Cama A, Garre ML, Rossi A, Andreussi L: Ependymomas of the posterior cranial fossa: CT and MRI findings. Neuroradiology 1995;37:238-243.

19 Tortori-Donati P, Fondelli MP, Rossi A, Cama A, Caputo L, Andreussi L, Garre ML: Medulloblastoma in children: CT and MRI findings. Neuroradiology 1996;38:352-359. 